\title{
The Title of This Paper Should Be in Title Case (See Below), Single Spaced, Times New Roman, Bold 14-point, and the Text Should Be Left Justified
}

First Author $^{1}$, Second Author ${ }^{2}$ and Third Author ${ }^{3}$ (Times New Roman 12-pt left justified). Each name should have a superscript number to reference the author's affiliation. Use single line spacing and leave one blank line between the title and author list and one blank line after the author's names before the list of affiliations.

1. Affiliation of the first author, including Department/Institution, City and Country. (12pt)

2. Affiliation of the second author, including Department/Institution, City and Country. (12pt)

3. Affiliation of the third author, including Department/Institution, City and Country. (12pt), leave one blank line after author's affiliations.

All papers should be a condensed version of your presentation and include all significant findings. Write the text so that readers who are not specialists can appreciate the purpose of the study and understand the procedures and conclusions. The text, entirely written in English, should include a brief introduction and motivation of the study, including experimental procedures, main results, and conclusions. It is not necessary to divide the text into sub-sections, except for the References section.

Capitalize all major words in your paper title as shown in the example above (nouns, pronouns, verbs, adjectives, adverbs, and some conjunctions); use lowercase for articles: the, a, and an; for the following conjunctions: and, but, for, or, and nor; and for the part of a proper name that would be lowercased in the text, such as de or von; as well as for the second part of a species name, such as fulvescens in Acipenser fulvescens, even if it is the last word in a title or subtitle.

Your paper must be submitted as Adobe PDF format with NO document security and the .pdf file name extension, AND as an editable word processing file (preferably Microsoft Word) in .doc, .docx, .txt, or .ltx formats. The PDF of the paper MUST have fonts and images embedded. Use a 12-point Times New Roman typeface and single spacing between lines. Use italics for taxonomic terms; do not use underlines. Avoid individualized formatting and special typefaces. Document margins should be one inch $(25.4 \mathrm{~mm})$ top and bottom and 0.75 inches $(19.1 \mathrm{~mm})$ left and right. Papers shall have a length of two (2) pages and must conform to these layout requirements; those with fewer pages will be rejected.

Skip a line before each new paragraph, but do not indent paragraphs. Page 1 should contain text only, using fully justified formatting, with no figures.

Page 2 may contain figures, figure captions, and tables in addition to text. Be sure to include figure labels and scale markers on all figures. Captions should be placed below all figures and tables. Artwork and tables must be electronically inserted into the document. Color figures may be used and will be reproduced in any electronic proceedings distributed. Printed copies, if made available, will be in grayscale, so authors should insure that color coding used for legends is distinguishable in grayscale. Line art must be created either in a drawing program or scanned into a suitable format for importing into the document. Check that the width of lines and size of fonts allow the figure to be clear at its final size.

References should be included at the end of the text, on page 2, with the appropriate reference styles for conference proceedings [1], journal articles [2], and books [3]. Indicate references in the text with 
Arabic numbers in square brackets, preferably at the end of the sentence, before the period. For four or more authors, use first-named author followed by "et al". Acknowledgments should be included in the last reference, which should be cited at the end of the last sentence of the text.

All submissions are reviewed for scientific content by the Program Committee. Contributions will be reviewed according to the following criteria: (a) relevance to a specific symposium, (b) scientific content, quality and innovative proposals, (c) clarity of the text and (d) compliance with the format. Papers not meeting the criteria above will not be accepted. All submissions for society and meeting awards, scholarships and prizes must meet these requirements. After the closing date for paper submissions, neither paper content nor author lists will be accessible for corrections.

All accepted papers must be presented by a meeting registrant. If the registrant cannot attend due to unforeseen circumstances an alternate presenter should be arranged by the submitter.

Presentations can be either platform or poster. The authors may express their preference about the form of presentation; however the Executive Program Committee reserves the right to make the final decision concerning all requests for platform presentations. All requests for poster presentations will be honored [4].

References: (Times New Roman, 12-pt, left justified)

[1] F Author et al, Conference Proceedings (year) p. 1.

[2] F Author, S Author and TH Author, Journal volume (year), p. 1.

[3] F Author in "Introduction to abstract writing", ed. D Writers, (Publisher, City) p.1.

[4] The authors acknowledge funding from the United Federation of Planets, X File Department, Grant Number 42. Dr. W Ho is thanked for his many useful discussions and contributions to this work.
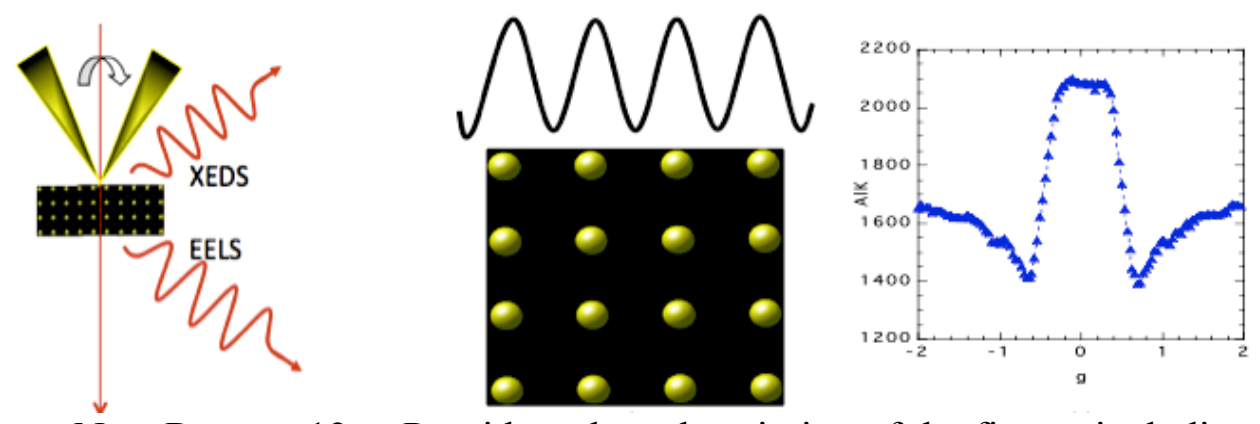

Figure 1. Times New Roman, 12pt. Provide a short description of the figure, including labels and scale markers as appropriate

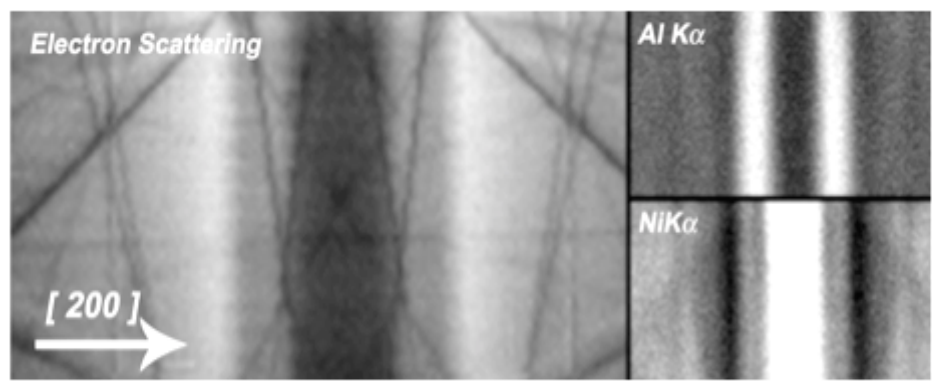

Figure 2. Times New Roman 12pt. Provide a short description of the figure, including labels and scale markers as appropriate. 